\title{
Evolución de la cirugía: ¿estamos preparados para romper paradigmas?
}

Evolution of surgery: care we prepared to break paradigms?

Evolução da cirurgia: ¿estamos preparados para quebrar paradigmas?

La cirugía, sin duda alguna, ha jugado un papel fundamental en la evolución de la medicina moderna: por medio de sus avances, la calidad de los tratamientos médicos ha mejorado de manera trascendental la expectativa de vida de muchas patologías. Algunos autores afirman que sus inicios se remontan a la época de la prehistoria, cuando tribus indígenas ya realizaban procedimientos quirúrgicos como amputaciones, litotomías, ablación de cataratas y trepanaciones de cráneos, entre otros. Sin embargo, su punto de inflexión se marco hace aproximadamente 150 años con el descubrimiento de la anestesia y la posterior aparición del uso de antibióticos (1).

Desde procedimientos con grandes incisiones conocidos como "more radical/major surgeries" en la década de 1950, hasta otros sin incisiones denominados "incisionless" a finales de los años 2000, los cambios que se han experimentado durante las últimas cinco décadas han sido revolucionarios. El acceso a mejores tecnologías diagnósticas e instrumentales quirúrgicos más avanzados ha permitido el desarrollo de nuevas técnicas quirúrgicas con abordajes mínimamente invasivos, incluyendo, entre ellos, la cirugía laparoscópica, la cirugía endoscópica y la cirugía robótica, todas ellas perteneciente al gran componente de cirugía mínimamente invasiva (MIS), por sus siglas en inglés, concepto inicialmente introducido en 1987 por el cirujano británico, John E.A Wickham. Entre sus ventajas se destacan: menor dolor postoperatorio, menor número de incisiones quirúrgicas, menor tiempo estancia hospitalaria, mejores tiempos de recuperación posquirúrgica, menores tasas de complicaciones intraoperatorias y menor riesgo de infecciones postoperatorias $(1,2)$.

La cirugía laparoscópica se consolidó a finales de los años 80 con la incorporación de la video laparoscopia, tecnología que venia siendo 
desarrollada desde la década de 1960 por varios grupos de ginecólogos y urólogos en Alemania. Su líder mas destacado y recordado es el Dr. Kurt Seemm. En la literatura médica, el primer caso de una cirugía laparoscópica exitosa fue reportado en 1987 por el cirujano francés Philip Mouret y se trató de una colecistectomía por laparoscopia. Dos años más tarde, el Colegio Americano de Cirujanos, (ACS), por sus siglas en inglés, dio el aval del nuevo procedimiento quirúrgico con el apoyo de otros líderes de la cirugía como los doctores Eddie Reddicky Douglas Olsen (2).

Este avance fue de tales proporciones, que los mismos principios técnicos fueron aplicados de manera paulatina a otros procedimientos quirúrgicos, los cuales hoy en día forman parte de la rutina del cirujano. De forma paralela, a inicios de la década de 1970, los doctores Shinya y Wolfe reportaban las primeras experiencias de remoción de pólipos colónicos utilizando colonoscopios rígidos; procedimientos caracterizados por tener una mínima morbilidad y mortalidad. Estos fueron los inicios formales de una nueva era llamada "cirugía endoscópica". Con el paso del tiempo dicha práctica se estableció sólidamente y de manera rutinaria en muchos centros de cirugía gastrointestinal al rededor del mundo.

Hace casi dos décadas, usando los mismos principios, se estableció el concepto de cirugía endoscópica a través de orificios naturales, por sus siglas en inglés, "NOTES", y posteriormente, se desarrolló un número considerable de técnicas quirúrgicas utilizando la combinación de instrumental endoscópico y laparoscópico, con el objetivo de tener acceso a la cavidad abdominal sin cicatrices externas. Sus representantes más destacados fueron los doctores Kalloo y Kantsevoy, cuyos primeros reportes datan de los años 2001 y 2003. Estos conceptos tomaron relevancia cuando fueron aceptados científicamente por la Sociedad Americana de Cirujanos Gastrointestinales y (SAGES) y la Sociedad Americana de Endoscopistas Gastrointestinales (ASGE). Entre sus grandes logros se destacaron la apendectomía transoral, la colecistectomía transvaginal, nefrectomía transvaginal y la manga gástrica transvaginal. Sus limitaciones para estandarizar las técnicas limitaron su popularidad, sin embargo, fueron importantes para el perfeccionamiento de distintos procedimientos años después (3).

El avance de este campo fue liderado de manera inicial por el Dr. Inoue, quien colaboró con el desarrollo de otras técnicas mínimamente invasivas incluyendo las disecciones endoscópicas submucosas, resecciones endoscópicas de lesiones benignas, miotomías endoscópicas perorales, piloromiotomías endoscópicas perorales, estricturoplastias endoscópicas en intestino delgado y colon, y divercitulotomias endoscópicas. Todos estos procedimientos se realizan de manera segura en la actualidad, con excelentes resultados clínicos a lo largo del mundo (3).

La cirugía robótica surgió con el objetivo de ofrecer diversas ventajas respecto a la cirugía laparoscópica tradicional, incluyendo la visión tridimensional, mayor destreza y movilidad, empleo de instrumentos articulados, mayor extensión de los movimientos, reducción del temblor y posición ergonómica del cirujano. La historia moderna inicia con el robot Puma 560, descrito en 1988 por el $D r$. Kwoh y utilizado para realizar biopsias neuroquirurgicas con mayor precisión. Para la cirugía gastrointestinal el gran paso se dio en el año 2001, cuando el Dr. Marecaux realizó la primera colecistectomía robótica en Nueva York, (EE.UU) utilizando el sistema quirúrgico ZEUS. Posteriormente, el sistema quirúrgico da Vinci se posesionó como el más completo y más desarrollado, contando con el aval de la Agencia Federal de Administración de Alimentos y Medicamentos de los Estados Unidos (FDA), por sus siglas en Ingles. Su uso no se ha limitado al campo de la cirugía general; urólogos, ginecólogos, cirujanos cardiovasculares y otras especialidades han tomado partida de las ventajas de estos sistemas quirúrgicos (4).

Finalmente, el futuro de la cirugía estará influenciado por varios factores: la persistencia de patologías quirúrgicas que se puedan prevenir con tecnologías modernas como modificaciones genómicas e inteligencia artificial, el desarrollo de nuevos avances que incluyen el uso de micro robots y nanotecnologías para crear técnicas quirúrgicas más eficaces, así como nano sensores para implementar diagnósticos tempranos que evitarán manejos quirúrgicos. El campo de la investigación avanza a pasos agigantados con el fin de proveer al paciente los mejores desenlaces clínicos posibles, trayendo consigo el reto de romper paradigmas, pues esos cambios en el futuro estarán sujetos a dilemas éticos y retos de aceptación por parte de sociedades científicas y académicas (5).

Juan S. Barajas-Gamboa, MD Clinical Research Fellow Digestive Disease Institute: Cleveland Clinic Abu Dhabi. Abu Dhabi, United Arab Emirates. 
UNAB Evolución de la cirugía: ¿estamos preparados para romper paradigmas?

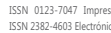

\section{Referencias}

1. Hindle KS, and Hindle SJ. A History of Surgery. J R Soc Med. 2001 Aug; 94(8): 423.

2. Kelley WE Jr1. The evolution of laparoscopy and the revolution in surgery in the decade of the 1990s. JSLS. 2008 Oct-Dec;12(4):351-7.
3. Litynski GS. Endoscopic surgery: the history, the pioneers. World J Surg. 1999 Aug;23(8):745-53.

4. Lane T. A short history of robotic surgery. Ann R Coll Surg Engl. 2018 May; 100(6 sup): 5-7

5. Alderson D. The Future of Surgery BJS. 2019;106:9-10. 